Marquette University

e-Publications@Marquette

Physics Faculty Research and Publications

Physics, Department of

6-1-2010

Simulations of Field Driven Domain Wall Interactions in Ferromagnetic Nanowires

Andrew Kunz

Marquette University, andrew.kunz@marquette.edu

Eric W. Rentsch

Marquette University

Published version. IEEE Transactions on Magnetics, Vol.46, No. 6 (June 2010), DOI. (C) 2010 Institute of Electrical and Electronics Engineers. Used with permission. 


\title{
Simulations of Field Driven Domain Wall Interactions in Ferromagnetic Nanowires
}

\author{
Andrew Kunz and Eric W. Rentsch \\ Physics Department, Marquette University, Milwaukee WI 53233 USA
}

\begin{abstract}
The interaction of domain walls in a single ferromagnetic nanowire has been observed with micromagnetic simulation. Domain walls separating domains of opposite magnetization move towards each other when an external field is applied along the long axis of the wire resulting in a collision. The final magnetic state of the wire after the collision will contain either zero (domain wall annihilation) or two (domain wall conservation) domain walls. Here we explore the behavior that determines the final state, showing that it depends on the initial domain wall configuration, the speed the domain walls are moving with before the collision, and the dimensions of the nanowire. A model is also presented which helps to determine the repulsive force the conserved domain walls exert on each other.
\end{abstract}

Index Terms-Magnetic domains, magnetic fields, micromagnetic simulation.

\section{INTRODUCTION}

$\mathbf{T}$ HE MOTION of a magnetic domain wall through a thin, narrow nanowire is a topic of considerable interest due to the numerous potential applications in data storage, logic operations, and sensing [1], [2]. Many of these devices require the presence of multiple domain walls, each of which may need to be independently controlled and moved. In long nanowires the magnetization of the wire is essentially aligned along the long axis of the wire and in the simplest case a transverse domain wall separates domains oppositely aligned in the wire as demonstrated in the cartoon Fig. 1(a) at time $t=0$ [3]. When an external field is used to move a domain wall the domain oriented in the direction of the applied field grows at the expense of the oppositely oriented domain as shown in the cartoon diagram in Fig. 1(a) at some time $t>0$.

In Figs. 1(b), (c) two domain walls exist in the wire and the central domain is oriented in the opposite direction to the externally applied field. In this field the central domain shrinks as time passes while the two outer domains grow-resulting in the motion of the domain walls towards each other [4]. Eventually the two domain walls collide with each other. In the simplest interpretation, the final magnetic state of the wire depends on the relative orientation of the magnetic moments of the colliding domain walls [5], [6]. When the magnetic moments of the two domain walls are in the same direction, as demonstrated in Fig. 1(b), the magnetic moments of the central domain rotate into the direction of moments of the domain walls during the collision leading to a fast reversal of the central domain and an annihilation of the two walls. If the two domain walls have magnetic moments oriented oppositely as demonstrated in Fig. 1(c) the rotation of the central domain is frustrated and the domain walls may repel as they get close to each other, potentially leading to a final magnetic state of the wire with two closely packed domain walls, called a $360^{\circ}$ domain wall. In this work we investigate the wire dimensions where the domain walls are expected to be preserved. In doing so we report that the field used to drive the walls together also plays a role in the preservation or annihilation of the domain walls. We describe

Manuscript received October 29, 2009; revised January 08, 2010; accepted January 12, 2010. Current version published May 19, 2010. Corresponding author: A. Kunz (email: andrew.kunz@marquette.edu).

Digital Object Identifier 10.1109/TMAG.2010.2041043

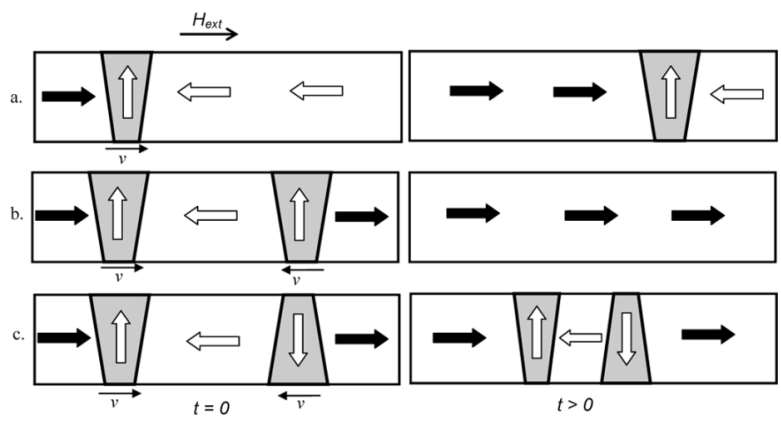

Fig. 1. Cartoon sketches of the relevant motion of a field driven domain wall as time passes. The domain in the direction of the field grows driving the left wall to the right every time. The walls on the right move to the left and in $b$. the walls annihilate and in c. they repel.

this behavior by finding the interaction energy of the walls as a function of their separation which has a maximum value. When the energy of a moving domain wall is greater than the maximum value, annihilation occurs.

Here we explore the case where the two domain walls have oppositely aligned magnetization and therefore the potential to remain in the wire after the collision to understand the properties which lead to domain wall conservation and to model the interaction and the force that the walls apply on each other.

\section{Simulation Details}

We use micromagnetic simulation techniques to explore the interactions of domain walls during the collision and to visualize the final magnetic state of the wire. The simulations follow the standard Landau-Lifshitz equation of motion for the three-dimensional rotation of the magnetic moments $m$ in the wire

$$
\frac{\partial \vec{m}}{d t}=-\gamma(\vec{m} \times H)-\frac{\alpha \gamma}{M_{s}} \vec{m} \times(\vec{m} \times \vec{H})
$$

with gyromagnetic ratio $\gamma=2.2 \times 10^{5} \mathrm{~m} /($ As) [7]. The total magnetic field $H$ includes the external field as well as the dipole and exchange fields with materials parameters for permalloy including saturation magnetization $M_{s}=8 \times 10^{5} \mathrm{~A} / \mathrm{m}$, and exchange constant $A=1.3 \times 10^{-11} \mathrm{~J} / \mathrm{m}$ with no crystalline anisotropy. We simulate wires with a minimum length of $4 \mathrm{mi}-$ crons and a varying rectangular cross-section. The wire is discretized into identical $4 \mathrm{~nm}$ cubes, integrated with a 4 th order predictor corrector technique with a simulated integration time 


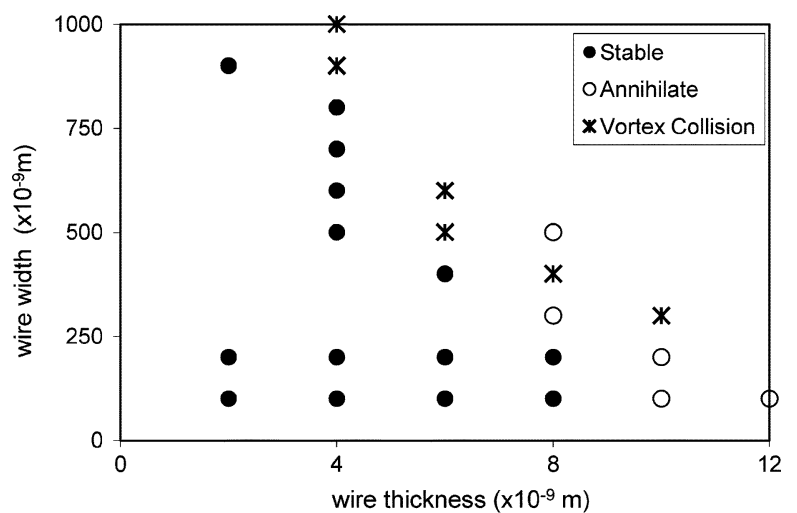

Fig. 2. Final magnetic state of the wire is presented as a function of the wire width and thickness. The solid dots correspond to a final state with two domain walls and the rest have zero domain walls. The open circles and the crosses correspond to different reversal mechanisms.

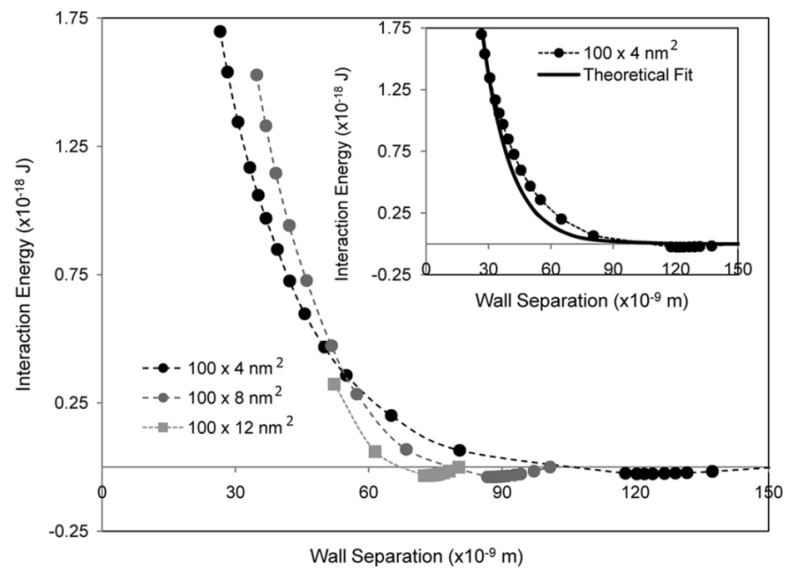

Fig. 3. Potential energy of the two domain walls as a function of their separation. As the walls are moved together from their equilibrium separation the potential energy increases rapidly as the walls repel. The inset shows a theoretical fit which models the repulsion as that of a solid sphere and a standard magnetostatic dipole attraction.

step of less than a picosecond and a damping parameter of $\alpha=$ 0.008 .

\section{RESULTS AND DISCUSSION}

Two domain walls of opposite orientation begin at the ends of the wire and are driven toward each other with a 10 Oe field. A field of 10 Oe, smaller than the Walker breakdown field, is chosen so that the domain walls travel quickly and with a constant spin configuration [8]. The final magnetic state of the wire is summarized in Fig. 2 as a function of the width and thickness of the wire. When the two domain walls remain in the wire we consider the structure stable. When the domain walls annihilate at the point of the collision we call that annihilation. A third, more complex, possibility also arises which we call a vortex state. In the thickest and widest wires the collision often forms a vortex domain wall in the wire which can be a long lasting configuration even though it is always eventually driven out of the wire (at the ends). The vortex state is equivalent to annihilation but the reversal dynamics are different which is why we separate it out. A good visualization of the vortex reversal mechanism has been recently published [9].

The probability that the domain walls annihilate during the collision increases as the wire thickness increases. As the thickness increases the shape anisotropy which holds the magnetization in the plane of the wire decreases. This opens up a new path for the reversal of the central domain by rotation out of the plane of the wire. The simple annihilation process always appears to take place with a rotation of the domain walls out of the plane of the wire. For all wires thicker than $12 \mathrm{~nm}$ thick the walls always annihilate. We find less of a dependence on the wire width as the thinnest wires simulated can be over a micron in width and still maintain two domain walls after the collision.

Fig. 3 shows the normalized energy as a function of the separation of the domain wall. We have normalized the interaction potential energy such that the interaction goes to zero as the walls are far apart. To create this plot we applied a 4 Oe field to the wire moving the domain walls together. This field ensured that the domain walls did not annihilate when driven together as is discussed below. The field was then turned off and the spins relaxed until the equilibrium $360^{\circ}$ wall state was found. The separation of the domain walls is then calculated. Because the domain walls are simple transverse walls we estimate the domain wall separation $r$ from the simulation as follows:

$$
r=\frac{L}{2}\left(1-\frac{M_{x}}{M_{s}}\right)
$$

where $L$ is the length of the wire, and the ratio of $M_{x}$ to $M_{s}$ is the reduced long axis magnetization of the wire. The domain wall separation has been verified by locating the positions along the wire where the magnetization along the long axis changes. The positions along the wire where the longitudinal magnetization changes would be the center of each wall. We find good agreement with the two techniques; however in our simulations we have immediate access to the magnetization of the wire so this technique is preferred.

The $360^{\circ}$ domain wall is a robust state under compressive fields. To generate the interaction energy of the domain walls with separation less than the equilibrium separation compressive external fields of increasing strength were applied. The maximum energy plotted corresponds with the largest applied field for which the domain walls remained in the wire. The height of the barrier is found to decrease as the wire thickness increases. Under a compressive external field, micromagnetic simulation showed that an 1800 Oe field was necessary to destroy the $360^{\circ}$ domain wall in a $4 \mathrm{~nm}$ thick wire whereas a 300 Oe field was sufficient in the $12 \mathrm{~nm}$ thick wires.

Magnetic fields were also placed along the long axis of the wire to separate the domain walls. Fig. 3 shows a weak and decreasing attraction between the domain walls as they move apart from their equilibrium separation. We approximate the domain walls as magnetic dipoles, each with magnetization perpendicular to their separation such that the dipole energy can be written as

$$
U_{\text {dip }}(r)=\frac{\mu_{0}}{4 \pi}\left(\frac{\vec{m}_{1} \cdot \vec{m}_{2}}{r^{3}}\right)
$$

where $\mu_{0}=4 \pi \times 10^{-7} \mathrm{~N} / \mathrm{A}^{2}$. The magnetic moments, $m=$ $M_{s} V$, of the two walls are equal as each wall encompasses the same volume $V$ in the wire. The wire width and thickness determine two dimensions and the domain wall length can be calculated from simulation results [10]. As the thickness of the wire increases it is seen in Fig. 3 that the strength of the attraction increases slightly. The increase in attraction follows from the increased strength of the magnetic moment of the domain wall 
due to the increase in volume of the domain wall due to the increased thickness.

The potential energy of the two domain walls increases rapidly as the walls are brought closer which implies a strong repulsion. During compression we model the repulsion similar to that of a solid sphere with a quickly dying exponential

$$
U_{\text {repel }}(r)=U_{0} e^{-r / \rho}
$$

where $U_{0}$ is the maximum value of potential and $\rho$ determines how fast the exponential decays. Using these expressions the total interaction energy can be estimated as

$$
U_{\text {tot }}(r)=U_{0} e^{-r / \rho}-\frac{\mu_{0} m^{2}}{4 \pi r^{3}} .
$$

By choosing appropriate values of $U_{0}$ and $\rho$ we were able to fit the measured interaction with this model. A fit for the $100 \times 4$ $\mathrm{nm}^{2}$ wire with $U_{0}=1.26 \times 10^{-17} \mathrm{~J}$ and $\rho=1100 \mathrm{~nm}$ is shown in the inset of Fig. 3. Finding the force the walls exert on each other as a function of their separation is a simple exercise involving taking the derivative of the potential energy function above as a function of the separation $r$.

In Fig. 2 we report that the domain walls annihilated during the collision in a $100 \times 12 \mathrm{~nm}^{2}$ wire, but in Fig. 3 we explore the interaction potential of the domain walls in this same wire showing a relatively large repulsion when the walls are close. This discrepancy can be explained by discussing the strength of the magnetic fields used to create the initial interaction. In Fig. 2 the walls were driven together with a 10 Oe field, but in Fig. 3 they were brought together using a 4 Oe field initially. The 10 Oe field immediately led to the destruction of the wall, where as the 4 Oe field created a stable $360^{\circ}$ domain wall which required a field of 300 Oe to be destroyed. We believe the reason for this behavior can be found in the kinetic energy of the wall. Because a transverse wall maintains its structure when driven by fields below the Walker breakdown field it is a simple procedure to track the location of each domain wall as a function of time with micromagnetic simulation. This allowed us to determine that when the walls were driven by a 10 Oe field each moved with a speed of roughly $350 \mathrm{~m} / \mathrm{s}$, where as a 4 Oe field moves the walls with a speed closer to $150 \mathrm{~m} / \mathrm{s}$ [11]. To test this behavior we ran interaction trials with varying applied fields. We calculated the domain wall speed and found the distance of closest approach of the domain walls. The results are shown in Fig. 4 and give the kinetic energy of the wall as a function of the applied driving field. When the wall kinetic energy is greater than the height of the barrier the walls annihilate and when the kinetic energy is less than the barrier height the walls bounce apart. The domain walls get closer to each other as the driving field and hence wall speed increase. The fact that there is a speed dependence to the domain wall interactions is not important in most cases because the field needed to create the wall speed necessary to overcome the barrier would be greater than the Walker field, meaning the wall changes structure and also moves slowly.

The interaction of transverse domain walls with opposite directions of magnetization has been studied by micromagnetic simulation. Antialigned transverse walls in narrow, thin nanowires exhibit a weak attraction when separated by a large

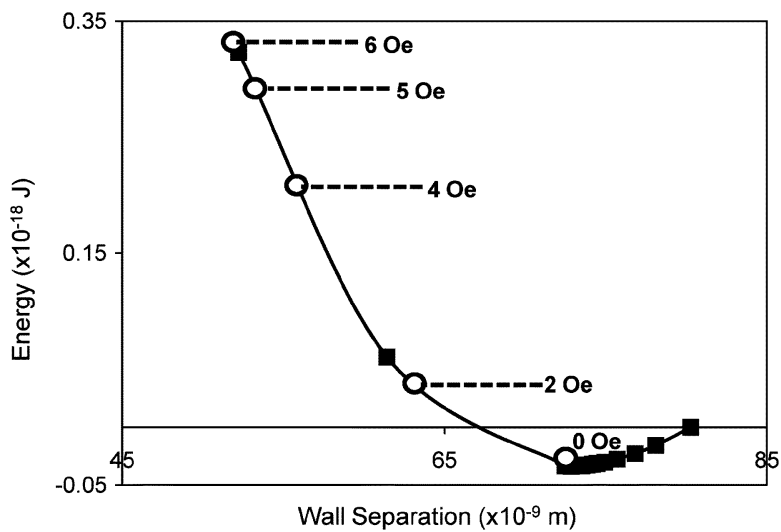

Fig. 4. Potential energy curve for the $100 \times 12 \mathrm{~nm}^{2}$ wire. The open circles represent the distance of closest approach for domain walls driven together by the listed fields. All greater fields led to annihilation as the wall kinetic energy was greater than the barrier height.

distance but a strong repulsion when they are brought close together ensuring that a collision between two such walls can preserve both walls if the wire is both thin enough and if the field driving them together is weak enough. Increasing the driving field leads to greater domain wall speeds which allows the walls to climb the barrier to reversal annihilating the walls or to reverse via nucleation of vortices if the field is greater than the Walker breakdown field [9]. The barrier height increases as the thickness of the wire decreases such that the domain walls are always preserved in collisions in thin wires.

\section{ACKNOWLEDGMENT}

This work was supported by the National Science foundation under Grant DMR-0706194.

\section{REFERENCES}

[1] S. S. P. Parkin, M. Hayashi, and L. Thomas, "Magnetic domain-wall racetrack memory," Science, vol. 320, pp. 190-194, 2008.

[2] D. A. Allwood, "Magnetic domain-wall logic," Science, vol. 309, pp. 1688-1692, 2005.

[3] R. D. McMichael and M. J. Donahue, "Head to head domain wall structure in thin magnetic strips," IEEE Trans. Mag., vol. 33, no. 5, pp. 4167-4169, 1997.

[4] J.-G. Zhu and Y. Zheng, "The Micromagnetics of Magnetoresistive Random Access Memory," in Spin Dynamics in Confined Magnetic Structures, I. B. Hillebrands and K. Ounadjela, Eds. Berlin Heidelberg: Springer-Verlag, 2002.

[5] A. Kunz, "Field induced domain wall collisions in thin magnetic nanowires," Appl. Phys. Lett., vol. 94, pp. 132502-, 2009.

[6] E.-M. Hempe, "Domain walls, domain wall transformations and structural changes in permalloy nanowires when subjected to current pulses," Phys. Stat. Sol. (a), vol. 204, no. 12, pp. 3922-3928, 2007.

[7] Micromagnetic Simulator, [Online]. Available: http://llgmicro.home. mindspring.com, v. 2.61

[8] N. L. Schryer and L. R. Walker, "The motion of $180^{\circ}$ domain walls in uniform dc magnetic fields," J. Appl. Phys., vol. 45, pp. 5406-5421, 1974.

[9] D. Djuhana, "Magnetic domain wall collision around the Walker breakdown in ferromagnetic nanowires," J. Appl. Phys., vol. 106, pp. 103926-, 2009.

[10] A. Kunz and S. C. Reiff, "Enhancing domain wall speeds in nanowires with transverse magnetic fields," J. Appl. Phys., vol. 103, pp. 07D903-, 2008.

[11] A. Kunz, "Simulating the maximum domain wall speed in a magnetic nanowire," IEEE Trans. Magn., vol. 42, no. 10, pp. 3219-3221, Oct. 2006. 